\title{
Anti-inflammatory and antiallergic effects of ketorolac tromethamine in the conjunctival provocation model
}

\author{
Andrea Leonardi, Fabiola Busato, Iva Fregona, Mario Plebani, Antonio G Secchi
}

\begin{abstract}
Aim-To study the effect of the topical anti-inflammatory drug, ketorolac, on (1) the clinical allergic reaction induced by the conjunctival provocation test (CPT); (2) the release of tryptase in tears; and (3) the expression of adhesion molecules on the conjunctival epithelium.

Methods-10 allergic but non-active patients were challenged in both eyes with increasing doses of specific allergen to obtain a positive bilateral reaction and rechallenged, after 1 week, to confirm the allergic threshold dose response. After 2 weeks, a third CPT was then performed bilaterally 30 minutes after topical application of ketorolac in one eye and placebo in the contralateral eye in a double blind fashion. Clinical symptoms and signs were registered $5,10,15$, and 20 minutes after challenge. The following objective tests were performed: tear tryptase measurement; tear cytology; and conjunctival impression cytology for immunohistochemical expression of ICAM-1 on epithelial cells.
\end{abstract}

Results-Compared with placebo, ketorolac significantly reduced the total clinical score and the itching score in the $20 \mathrm{~min}$ utes after challenge $(p<0.0005)$. Tear levels of tryptase were significantly reduced in the ketorolac pretreated eyes compared with placebo $(p<0.03)$. Eosinophils, neutrophils, and lymphocytes in tear cytology were significantly lower in ketorolac treated eyes compared with placebo. A significant difference in the epithelial expression of ICAM-1 was observed between placebo and ketorolac treated eyes $(\mathbf{p}<0.05)$.

Conclusion-Ketorolac proved to be effective in reducing mast cell degranulation, as indicated by significantly decreased tryptase tear levels, as well as the clinical and cytological allergic reaction.

(Br f Ophthalmol 2000;84:1228-1232)

Seasonal allergic conjunctivitis is a very common disease with symptoms such as itchy and tearing eyes, conjunctival redness, and chemosis and eyelid swelling. Ocular allergic inflammation is a consequence of single or repeated natural challenges to environmental allergens that induce IgE specific mast cell activation and the subsequent cascade of inflammatory mediators and cellular infiltration.
The allergic reaction induced by the specific conjunctival provocation test (CPT) reproduces exactly the signs and symptoms of the acute seasonal allergic reaction. In this model, a single episode is induced in a standardised manner allowing for a homogeneous baseline for all allergic patients in the study independent of the allergen used for challenge. ${ }^{2}$ The CPT reproduces both the immediate and late allergic ocular response. ${ }^{3}$ The early phase of this reaction is characterised by mast cell activation and the release of preformed and newly formed mediators such as histamine, tryptase, prostaglandins, and leukotrienes. ${ }^{4}$ Mast cell degranulation also induces activation of vascular endothelial cells and the expression of chemokines and adhesion molecules, such as ICAM-1/CD 54. ${ }^{5}$ These factors lead to a latent recruitment phase that ushers in the inflammatory late phase reaction characterised by cellular infiltration of the conjunctival mucosa. The conjunctival reaction induced by allergen challenge can be monitored in various ways, by scoring the clinical symptoms and signs, evaluating conjunctival or tear cytology, ${ }^{6}$ and/or measuring inflammatory mediators in the tear film. ${ }^{78}$

Ketorolac tromethamine $0.5 \%$ ophthalmic solution is a highly potent non-steroidal anti-inflammatory agent that decreases the synthesis of prostaglandins by inhibiting the enzyme, cyclo-oxygenase. The pharmacological activity of prostaglandins (PGs) accounts for many of the signs, symptoms, and events associated with experimental and clinical allergic conjunctivitis. ${ }^{9}$ In recent studies, the clinical efficacy of ketorolac in the treatment of seasonal allergic conjunctivitis has been evaluated. ${ }^{1011}$ The present study was performed to determine the protective effect of ketorolac on allergic conjunctival inflammation induced by allergen challenge. In addition to a clinical evaluation, the objective variables of tryptase tear levels, tear cytology, and ICAM-1 immunohistochemical expression were considered to better evaluate the anti-inflammatory effects of this drug.

\section{Materials and methods}

PATIENTS

Ten asymptomatic patients (age range 18-35 years; eight males, two females) with a clinical history of seasonal allergic conjunctivitis were included in this randomised, placebo controlled, double blind study. All patients were asymptomatic and reacted positively to the prick test (weal diameter $>3 \mathrm{~mm}$ ) or by radio- 
allergosorbent test (RAST) (class $>3$ ). The allergen that gave the greatest response by prick test and which was most clinically correlated with the seasonal symptoms was chosen for CPT: six patients were challenged with rye grass, three with Alternaria alternata, and one with Parietaria officinalis. The CPT was performed according to the standardised procedure described by Abelson et al. ${ }^{2}$

STUDY DESIGN

At visit 1, the allergen threshold dose that induced a positive conjunctival reaction was determined by challenging both eyes with one $20 \mu \mathrm{l}$ drop of allergen in serial dilutions (10, 50, 100, 200, 300 allergen unit RAST (AUR)/ $\mathrm{ml}$, increasing the dose every 15 minutes until a clinical reaction with a score of at least $2+$ itching and redness was obtained. Seven days later, a second challenge with the predetermined threshold dose was repeated to confirm the conjunctival reaction. After 2 weeks (visit 3; drug evaluation day), patients were given a single dose of ketorolac in one eye and placebo in the contralateral eye 30 minutes before CPT at the threshold dose.

CLINICAL AND LABORATORY EVALUATIONS

Conjunctival signs and symptoms (itching, burning/foreign body sensation, tearing, redness, chemosis, eyelid swelling) were assessed using a score of 0 (none) to 3 (severe) for each eye, before drug administration, before allergen challenge and 5,10,15, and 20 minutes after challenge.

Tear samples $(50 \mu \mathrm{l})$ were collected from both eyes with a capillary tube, 1 hour before $\mathrm{CPT}$ and within 10 minutes after CPT, and stored at $-20^{\circ} \mathrm{C}$ for tryptase measurement (RIA Pharmacia Upjohn, Uppsala, Sweden). The lower limit of sensitivity of the test was 1 $\mu \mathrm{g} / 1$.

Tear cytology was performed 30 minutes after CTP to determine inflammatory cell number. Tear samples $(2 \mu \mathrm{l})$ were collected and placed on precoloured slides (Testsimplets, Boeringher Mannheim). The number of neutrophils, eosinophils, basophils, and lymphocytes were immediately counted in five consecutive microscopic fields at $250 \times$ magnification power. Conjunctival impression cytology specimens were collected from both eyes at baseline on visit 1 before CPT and on visit 3, at 40 minutes after challenge using $0.22 \mu \mathrm{m}$ millipore filters. In each eye, one semilunar filter was applied without exerting pressure onto the superior bulbar conjunctiva. Membranes were removed immediately after contact, moistened in phosphate buffered saline (PBS pH 7.4), and firmly pressed onto a gelatin covered slide to transfer as many conjunctival cells as possible for immunohistochemistry.

CD54/ICAM-1 monoclonal antibody (Bender MedSystems, Switzerland) was used for immunohistochemical staining of conjunctival epithelial cells obtained by impression cytology. A sensitive immunoenzymatic alkaline phosphatase-monoclonal anti-alkaline phosphatase complex procedure was employed to detect ICAM-1 expression on epithelial cells

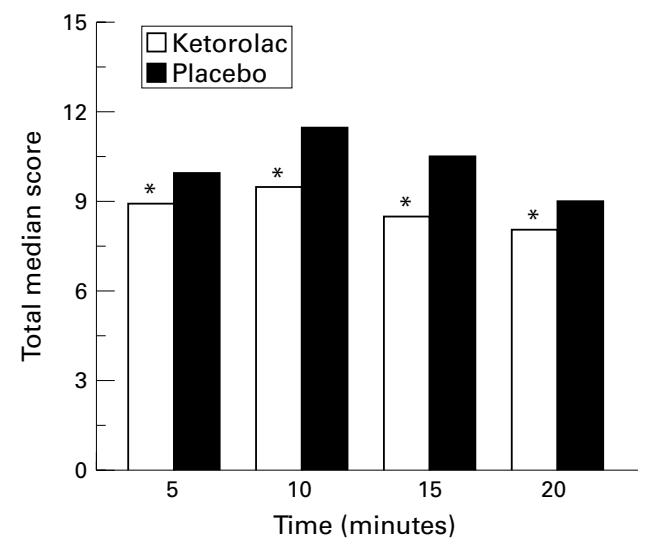

Figure 1 Total symptom score at 5, 10, 15, and 20 minutes after CPT and pretreatment with ketorolac tromethamine in one eye and placebo in the contralateral eye $(n=10)$. Ketorolac pretreatment showed a significant reduction in the time course of the total clinical reaction $\left({ }^{*} p<0.001\right.$ by Wilcoxon test $)$.

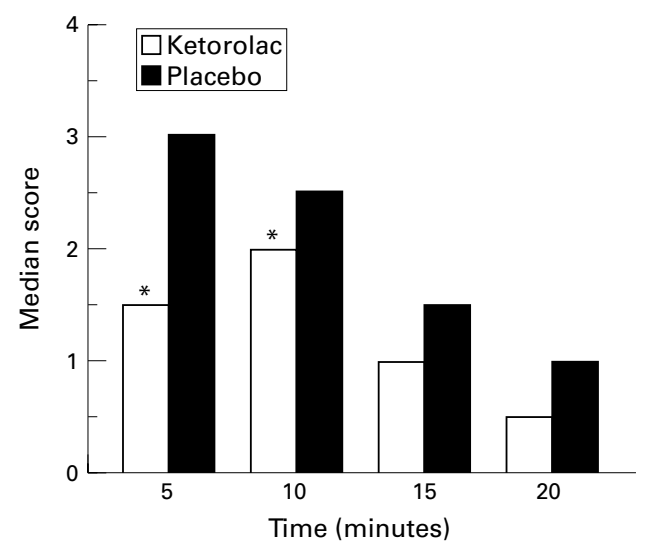

Figure 2 Time course of the symptom of itching after CPT and pretreatment with ketorolac tromethamine in one eye and placebo in the contralateral eye. Ketorolac pretreatment showed a significant reduction of itching at 5 and 10 minutes $\left({ }^{\star} p<0.05 ;{ }^{\star *} p<0.01\right.$ by Wilcoxon test).

according to Ciprandi et al. ${ }^{12}$ Slides were examined by three masked investigators. The positive red reaction in the epithelium was analysed in five representative fields $(\times 250)$ from the impression cytology specimens. It was classified as very intense $(3+)$, intense $(2+)$, slight $(1+)$, or absent (0).

\section{STATISTICAL ANALYSIS}

The non-parametric Wilcoxon signed rank test was used to examine the treatment effect of ketorolac versus placebo. The clinical variables considered were the sum symptom score within 20 minutes after CPT, and the score of each sign and symptom at each evaluation time and within the 20 minute time period. The same test was used to assess the difference in number of inflammatory cells, the score of ICAM-1 expression, and tryptase tear levels. The level of significance was fixed in the protocol as $\mathrm{p}<0.05$. Data are presented as median and minimum and maximum values.

\section{Results}

All the patients concluded the trial as planned. The reaction induced by CPT was symmetrical in both eyes and reproducible from visit 1 


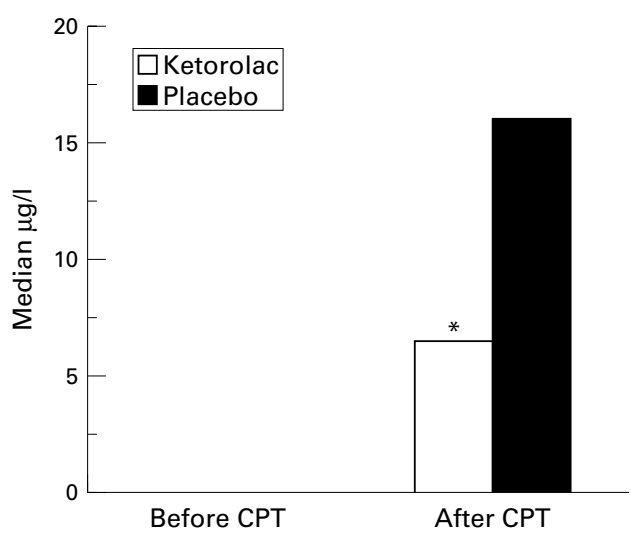

Figure 3 Median tryptase tear levels before and after CPT. Before challenge, levels were undetectable, while after challenge tryptase tear levels were significantly lower in eyes pretreated with ketorolac ( ${ }^{\star} p<0.05$ by Wilcoxon test).

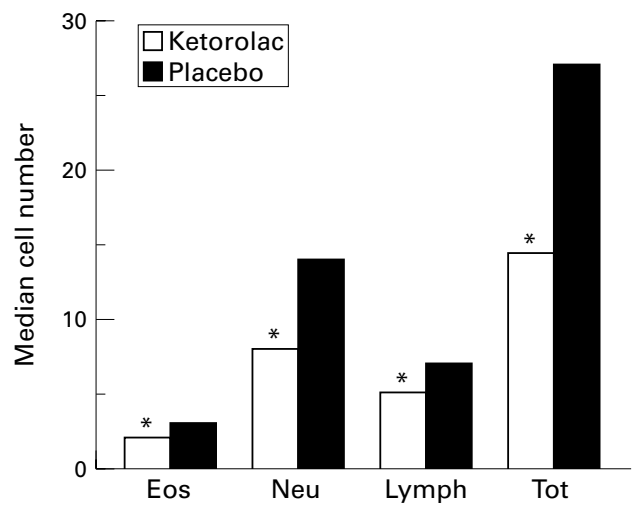

Figure 4 Tear cytology performed at 30 minutes after CPT. The number of eosinophils (eos), neutrophils (neu), lymphocytes (lymph), and the total number of inflammatory cells (tot) were significantly lower in the eyes pretreated with ketorolac ( ${ }^{*} p<0.05$ by Wilcoxon test).

to visit 2 -that is, no significant difference was found in the CPT score at visit 1 versus visit 2 . The allergic reaction induced, when considered as the total sum score of signs and symptoms at all evaluation time points, was significantly lower in the eyes pretreated with ketorolac compared with placebo (31.5 (range $20-47)$ versus $38(25-56) ; \mathrm{p}<0.0005)$. After ketorolac pretreatment, the total scores for itching and redness were significantly lower compared with that of the placebo (median itching: 5 (1-9) versus $8.5(2-12) ; \mathrm{p}<0.0005$; median redness: 8 (2-11) versus 9.5 (5-11); $\mathrm{p}<0.001)$. At each time point considered, the sum score of signs and symptoms was significantly lower in the ketorolac eyes $(p<0.0001)$ (Fig 1). The time course for itching was lower in the ketorolac treated eyes compared with placebo (Fig 2).

Median tryptase tear levels were significantly higher after CPT compared with baseline preCPT. In the eyes pretreated with ketorolac, tryptase tear levels were lower compared with those pretreated with placebo $(6.5(0-141) \mu \mathrm{g} / 1$ versus $16(0-65) \mu \mathrm{g} / 1 ; \mathrm{p}<0.03)$ (Fig 3).

Tear cytology performed 30 minutes after CPT showed a significant reduction in the number of inflammatory cells in the eyes pretreated with ketorolac compared with placebo: eosinophils: $2(0-5)$ versus $3(0-10)$,
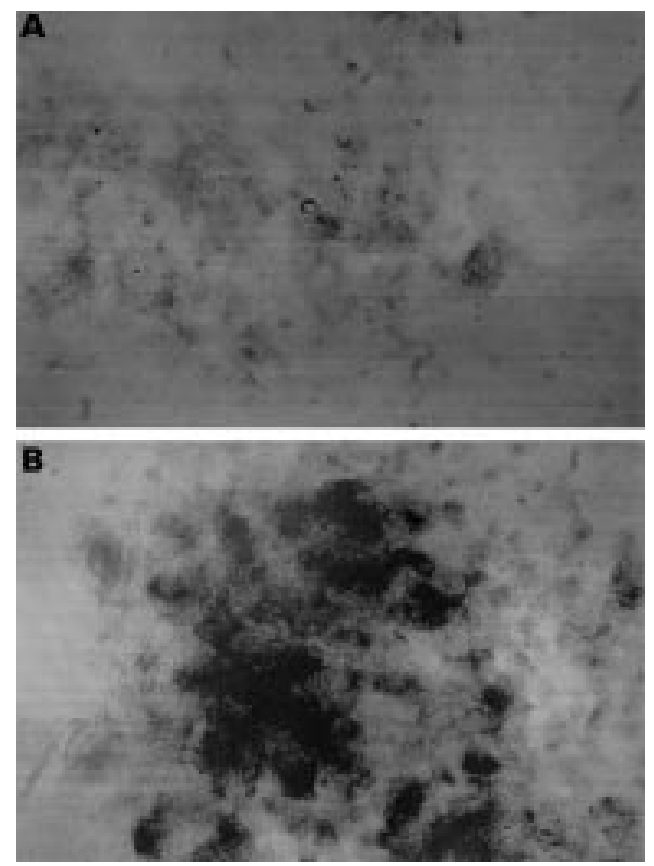

Figure 5 Immunohistochemical expression of ICAM-1 on conjunctival epithelial cells in one eye pretreated with ketorolac $(A)$ and in the contralateral eye pretreated with placebo (B). Note the lesser staining in $(A)$.

p<0.03; neutrophils: 8 (4-28) versus 14 (3-38), p<0.0001; lymphocytes: 5 (0-15) versus $7(0-17), \mathrm{p}<0.01$; total number of cells: 14.5 (4-39) versus 27 (3-51), p<0.0001 (Fig $4)$.

Expression of the inflammatory marker, ICAM-1, on epithelial cells was absent at baseline. After CPT on the drug evaluation day, ICAM-1 expression was significantly lower in eyes pretreated with ketorolac compared with those pretreated with placebo $(0.5(0-1)$ versus $1.5(0-3) ; \mathrm{p}<0.03)$ (Fig 5).

\section{Discussion}

The conjunctival provocation test (CPT) is a useful tool for the diagnosis of patients with suspected allergic conjunctivitis, ${ }^{13}$ for the study of the pathophysiology of the allergic reaction, ${ }^{3-8}$ and for the evaluation of the efficacy of antiallergic drugs. ${ }^{2}$ It reproduces in the physician's office the clinical signs and symptoms and inflammatory cascade of the allergic reaction in a standardised manner.

The clinical hallmark of allergic conjunctivitis is the subjective symptom of ocular itching. While it is nearly impossible to prove a drug effect on itching in a seasonal conjunctivitis study, the CPT model of allergy allows for the precise evaluation of this most important variable. Objective variables are needed, however, for a more thorough assessment of the pharmacological effects of therapeutic agents. Reliable cytological techniques and tear mediator assays are valid objective variables to be used in conjunction with CPT, the results of which increase the validity of the model in light of the difficulties of small sample size, timing of sample collection, and data reproducibility. The association of clinical symptomatology and multiple laboratory tests may lead to the 
most well rounded results for the evaluation of an antiallergic drug when the reaction is reproduced by CPT.

In the present study ketorolac was shown to have protective effects on conjunctival allergic inflammation induced by specific allergen challenge. The overall symptoms of ocular allergy and the main symptom of itching were significantly decreased by pretreatment with ketorolac tromethamine compared with placebo. Similar results were reported in environmental clinical studies in which ketorolac was proved to provide rapid relief from itching during seasonal allergic conjunctivitis. ${ }^{10}{ }^{11}$ Only the early phase of the reaction was considered in the present study since only threshold allergen doses which usually don't induce a clinically and cytologically relevant late phase were used for conjunctival challenge. ${ }^{3}$

Allergic inflammation is triggered by $\operatorname{IgE}$ mast cell activation and the subsequent release of preformed and newly formed mediators, such as histamine, tryptase, prostaglandins, leukotrienes, platelet activating factor (PAF), and cytokines, such as tumour necrosis factor alfa $(\mathrm{TNF} \alpha)$, interleukin 4 (IL-4), IL-5, IL-6 and IL-8. ${ }^{4}$ Histamine is rapidly released by mast cells but also rapidly inactivated by naturally occurring histaminase enzymes, which have been shown to be deficient in vernal conjunctivitis. ${ }^{14}$ The use of tryptase levels to assess mast cell activation has advantages compared with assessing levels of histamine, since tryptase is concentrated only in mast cells and its tear levels, after allergen provocation, peak within 10 minutes and return to baseline after 40 minutes. $^{15}$ In the present study, mast cell activation was proved to be reduced by ketorolac since tryptase tear levels were significantly lower in eyes pretreated with the drug. Reduced mast cell degranulation may result from a direct effect of ketorolac on mast cell membranes or from a reduced neurogenic inflammation and secondary activation of mast cells, since ketorolac has a potent effect on nociceptive nerve endings and subsequent axon responses. ${ }^{16}$

The reduced expression of ICAM-1 on epithelial cells was further evidence of a positive effect of ketorolac tromethamine as an antiinflammatory/antiallergic compound. In fact, the initial cellular inflammatory recruitment phase, driven by adhesion molecule expression, was reduced by ketorolac, as also shown by the significant reduction of inflammatory cells in tear cytology. The adhesion molecule ICAM-1/CD54, an immunoglobulin superfamily member, whose natural ligand is the $\beta 2$ integrin LFA-1 expressed on leucocytes, including eosinophils, is expressed on inflammed endothelium and epithelium in several diseases including allergic conjunctivitis. ${ }^{12}{ }^{17}{ }^{18}$ ICAM-1 expression on conjunctival epithelium was shown to be an early event (30 minutes after CPT) which persisted for up to 48 hours when a high allergen dosage was employed. It was related to clinical variables and inflammatory cell infiltration, ${ }^{12}$ and was induced in vitro by several cytokines. ${ }^{19}$ Ketorolac may have induced a downregulation of ICAM-1 expres- sion, acting directly on epithelial cells or, more indirectly, may have reduced mast cell activation and the release of soluble factors which upregulate ICAM-1 expression.

The products of arachidonic acid metabolism, PGs, and leukotrienes (LTs), have an important role in the development of the allergic ocular reaction. $\mathrm{PGD}_{2}$, the main prostaglandin produced by mast cells, was found in previous studies to increase in tears after allergen challenge, ${ }^{7}$ and when applied topically to the conjunctiva, to reproduce the signs and cytology of patients with allergic conjunctivitis. ${ }^{20}$ In an animal model of allergic conjunctivitis, $\mathrm{PGF}_{1}$ was detected in tears during early and late phase reactions, ${ }^{21}$ while $\mathrm{PGE}_{2}$ and $I_{2}$ were found to be potent pruritogenic substances in the conjunctival tissue. ${ }^{9}$ Discrepancies in leukotriene tear levels after challenge have been reported, ${ }^{722}$ but topical application of LTs have been shown increase vascular permeability ${ }^{23}$ and eosinophil chemotaxis. ${ }^{24}$

Ketorolac tromethamine is a potent nonsteroidal anti-inflammatory agent that has been found to be 180 times as potent as aspirin, inhibiting the activity of cyclooxygenase and suppressing the production of prostaglandins and other arachidonic acid metabolites. ${ }^{16}$ Topical ketorolac was found to be effective in the treatment of ocular inflammation ${ }^{25}$ and allergic conjunctivitis. ${ }^{10} 11$ Although in the present study the allergic reaction was monitored by the assessment of other inflammatory markers, the inhibitory effect of ketorolac on PG production was indirectly demonstrated by the reduction of itching and cellular chemotaxis.

In conclusion, a significant therapeutic effect of ketorolac tromethamine on conjunctival induced allergic reaction has been proved clinically, by the reduction of itching, redness, and overall symptoms and, for the first time, objectively, by the reduction of mast cell tryptase in tears, ICAM-1 expression on epithelial cells, s and tear cytology.

The authors have no financial interest in the product evaluated in this study.

1 Abelson MB, Schaefer K. Conjunctivitis of allergic origin: mmunologic mechanisms and current approaches to therapy. Surv Ophthalmol 1993;38(Suppl):115-32.

2 Abelson MB, Chambers WA, Smith LM. Conjunctival allergen challenge. A clinical approach to studying allegic conjunctivitis. Arch Ophthalmol 1990;108:84-8.

3 Bonini S, Bonini S, Bucci MG, et al. Allergen dose response and late symptoms in a human model of ocular allergy. $\mathcal{f}$ Allergy Clin Immunol 1990;86:869-76.

4 Galli SF, Costa JJ. Mast-cell-leukocyte cytokine cascades in allergic inflammation. Allergy 1994;24:203-6.

5 Canonica GW, Ciprandi G, Pesce GP, et al. ICAM-1 on epithelial cells in allergic subjects: a hallmark of allergic inflammation. Int Arch Allergy Immunol 1995;107:99-102.

6 Bonini S, Bonini S, Vecchione M, et al. Inflammatory changes in conjunctival scrapings after allergen provocation in humans. F Allergy Clin Immunol 1988;82:462-9.

7 Proud D, Sweet J, Stein P, et al. Inflammatory mediator release on conjunctival provocation of allergic subjects with allergen. F Allergy Clin Immunol 1990;85:896-905.

8 Leonardi A, Smith LM, Fregona IA, et al. Tear histamine and histaminase during the early (EPR) and late (LPR) phases of the allergic reaction and the effects of lodoxamide. Eur f Ophthalmol 1996;6:106-12.

9 Woodward DF, Nives AL, Hawley SD, et al. The pruritogenic and inflammatory effects of prostanoids in the
conjunctiva. F Ocular Pharmacol Ther 1995;11:339-348.

10 Raizman MB. Results of survey of patients with ocular Raizman MB. Results of survey of patients with ocular
allergy treated with topical ketorolac tromethamine. Clin Therapeutics 1995;17:882-90.

11 Ballas Z, Blumenthal M, Tinkelman DG, et al. Clinical evaluation of ketorolac tromethamine $0.5 \%$ ophthalmic 
solution for the treatment of seasonal allergic conjunctivitis. Surv Ophthalmol 1993;38(Suppl):141-8.

12 Ciprandi G, Buscaglia S, Pesce GP, et al Allergic subjects express intercellular adhesion molecule 1 (ICAM-1 or CD54) on epithelial cells of conjunctiva after allergen challenge. F Allergy Clin Immunol 1993;91:783-92.

Battista C, Gismondi M, et al. Antigen sensitivity evaluated by tear and serum IgE, skin tests, and conjunctival and nasal provocation tests in patients with allergic eye diseases. Eye 1993;7:461-4.

14 Abelson MB, Leonardi A, Smith LM, et al. Histaminase activity in patients with vernal keratoconjunctivitis. $O p h-$ thalmology 1995;102:1958-63.

15 Butrus SI, Ochsner KI, Abelson MB, et al. The level of tryptase in human tears. An indicator of activation of conjunctival mast cell. Ophthalmology 1990;97:1678-83.

16 Rooks WH, Maloney PJ, Shott LD, et al. The analgesic and anti-inflammatory profile of ketorolac and its tromethamti-inflammatory profile of ketorolac and

17 Bacon AS, McGill JI, Anderson D, et al. Adhesion molecules and relationship to leukocyte levels in allergic eye disease. Invest Ophthalmol Vis Sci 1998;39:322-30

18 Cook EB, Sthal JL, Miller ST, et al. Isolation of human conjunctival mast cells and epithelial cells: tumor necrosis factor- $\alpha$ from mast cells affects intercellular adhesion ecule 1 expression on epithelial cells. Invest Ophthalmol Vis Sci 1998;39:336-43.
19 Paolieri F, Battifora M, Riccio AM, et al. Intercellular adhesion molecule-1 on cultured human epithelial cell lines: influence of proinflammatory cytokines. Allergy 1997;52: 521-31

20 Abelson MB, Madiwale NA, Weston JH. The role of prostaglandin $\mathrm{D}_{2}$ in allergic ocular disease. In: O'Connor GR, Chandler JW, eds. Advances in immunology and immunopathology of the eye. New York: Masson, 1985:163-6.

21 Helleboid L, Khatami M, Wei ZG, et al. Histamine and prostacyclin. Primary and secondary release in allergic conjunctivitis. Invest Ophthalmol Vis Sci 1991;32:2281-9.

22 Bisgaard $\mathrm{H}$, Ford-Hutchinson AW, Charleston S, et al. Detection of leukotriene $\mathrm{C}_{4}$-like immunoreactivity in tear fluid from subjects challenged with specific allergen. Prostaglandins 1984;27:396.

23 Gary RK, Woodward DF, Nieves AL, et al. Characterization of the conjunctival vasopermeability response to leukotriens and their involvement in immediate hypersensitivity. Invest Ophthalmol Vis Sci 1988;29:119-26.

24 Trocme SD, Gilbert MC, Allansmith MR, et al. Characteristics of the cellular response of the rat conjunctiva to tics of the cellular response of the rat conjunctiva to topically

25 Flach AJ, Dolan BJ, Irvine AR. Effectiveness of ketorolac tromethamine $0.5 \%$ ophthalmic solution for chronic aphakic and pseudophakic cystoid macular edema. $\mathrm{Am} \mathcal{f}$ Ophthalmol 1987:103:479-86. 